\title{
Teaching Fourier Series Expansions in Undergraduate Education with the Help of the FouSE Android Application
}

\author{
http://dx.doi.org/10.3991/ijim.v8i1.3455 \\ M.J.C.S. Reis ${ }^{1}$, S. Soares ${ }^{1}$, S. Cardeal $^{2}$, R. Morais ${ }^{1}$, E. Peres ${ }^{1}$ and P.J.S.G. Ferreira ${ }^{3}$ \\ ${ }^{1}$ University of Trás-os-Montes e Alto Douro, Vila Real, Portugal \\ ${ }^{2}$ Portugal Telecom Inovação, Aveiro, Portugal \\ ${ }^{3}$ University of Aveiro, Aveiro, Portugal
}

\begin{abstract}
This paper discusses the teaching of Fourier Series concepts in undergraduate Electrical Engineering education with the help of an Android application, especially developed to that end. In order to better understand the links and implications, some of the basic Fourier series theory is briefly reviewed. The Android application has an easy-to-use, friendly interface, conceived to help undergraduate students test and assess the Fourier series expansions on a typical set of signals. The application also allows the students to control the total approximation error and the number of terms/harmonics used in the expansions. It has been found a very useful learning resource in the Fourier series context.
\end{abstract}

Index Terms-Android application; Fourier series expansions; undergraduate Electrical Engineering education

\section{INTRODUCTION}

Fourier series expansions and Fourier theory in general, are among the most important studied topics in undergraduate Electrical Engineering education. Consequently and naturally, a large fraction of the signal processing and also mathematical literature is devoted to the teaching of Fourier series expansions, and Fourier theory in general. Having a solid understanding on the most basic principles of Fourier series expansions is of utmost importance, especially when dealing with problems which fall, for example, in the scope of multidimensional signal reconstruction, and which includes sampling theory, interpolation, extrapolation, signal and image conditioning, interactive image repair, deconvolution and other inverse problems, reconstruction in tomography, filter design, and much more.

We believe that a first course focusing on Fourier series expansions would benefit from the existence of a tool that let students test and assess Fourier series expansions, anytime and anywhere they want, and using their own Android powered devices (for example, during the lunch time conversations, using their own smartphones and tablets). Moreover, this application can be a welcome addition to the toolbox of Electrical Engineering students, especially to those interested in telecommunications, information theory, or signal/ image processing in general.

Solving problems while experimenting and comparing the available algorithms is of fundamental importance in the learning process. The application presented in this paper was aimed at helping students do that. Additionally, we felt a need for tools that could be easily used inside or outside the classroom, easily updated and/or maintained, and that the students could use on their own smartphones and tablets, taking advantage of the concept "anytime, anywhere" and the increasing number of available mobile devices with the required functionalities and capacities, and the number of students using them.

The next section, section II, is used to define "mobile learning" and introduce some concepts related to it. In section III the Fourier series definition used in the Android application is presented, along with a typical/traditional example, to help clarify the ideas that lead to the development of the application. In section IV the Android application is presented, and in section $\mathrm{V}$ we present and discuss some of the results achieve with the survey applied to the users (students) of the application. The paper ends with section VI, where some conclusions are presented.

\section{THE "MOBILE LEARNING" CONCEPT}

The objective of Mobile Learning (mLearning) is to provide the learner with the ability to learn anywhere and at any time. However, the term mLearning can have different meanings. It is closely related to eLearning, educational technology and distance education, but it is distinct in its focus on learning across contexts and learning with mobile devices.

One definition of mobile learning is "Any sort of learning that happens when the learner is not at a fixed, predetermined location, or learning that happens when the learner takes advantage of the learning opportunities offered by mobile technologies" [1]. Clark Quinn (2000, http://www.linezine.com/2.1/features/cq mmwi yp.htm) defines mLearning as "eLearning through mobile computational devices: Palms, Windows CE machines, even your digital cell phone". Harris, [2], defines mLearning as the intersection point between mobile computing and eLearning, producing a learning experience "anytime" and "anywhere".

mLearning is indeed characterized by this capacity of accessing learning resources from anywhere, at any time, with high search capacities, high interaction, high support for an effective learning and a constant valorization based on the performance. So, one can see mLearning as an extension of eLearning, but characterized by its capacity of being independent in terms of space and time. The idea is to have small devices capable of being linked to the 
internet, easy data input, and the ability to display high resolution images and also (very) good sound capacities. Hence, mLearning can benefit from all advantages of the web added to mobile technologies.

In summary, we may say that mLearning relies on the utilization of mobile technologies at the service of the processes associated with teaching and learning. In other words, with the use of mobile devices, learners can learn from various locations.

Like any other technology and methodology, care must be taken when designing and implementing mobile applications intended for mLearning. For example, [3] discusses the design of personal mobile technologies for lifelong learning. Reference [4] reports an open source document search engine and mobile architecture to provide mobile learning support for trainee social workers in the UK, also presenting and discussing its benefits and constraints. The authors of [5] discuss the development of smart adaptive user interfaces for mobile e-Business applications, and the lessons learned. Universal instructional design principles for mobile learning are presented and discussed in [6]. In [7], the modelling, design, development and evaluation of a hypervideo application for digital systems teaching is presented and discussed. The process of life-long learning, in conjunction with three different models for mobile/ubiquitous applications is discussed in [8]. A knowledge engineering approach to developing e-libraries to assist teachers in defining metadata in mLearning contexts is proposed in [9]. Reference presents a formative assessment-based mobile learning approach to improve learning attitudes and achievements of students in southern Taiwan, and describes a system to support the generation of adaptive mobile learning environments where students and teachers can accomplish different types of individual and collaborative activities in different contexts. The use of mobile devices and technology was also presented and discussed during the process of review, design, and delivery of a course in modern embedded systems, an international collaborative teaching project involving the University of New South Wales (Australia), Manchester University, and Imperial College, London University (United Kingdom) [12].

As far as we know, the use of mobile devices in mLearning contexts to teach Fourier series expansions in undergraduate Electrical Engineering courses was first proposed in [13]. Here, we present a new version of the application and discuss the results achieved so far.

\section{FOURIER SERIES DEFINITION}

We believe that a small introduction to Fourier series will help to clarify the ideas that lead to the development of the application, as well as the links and implications. This section is dedicated to this.

A signal (function) $s(t)$ is said to have period $T$ if $s(t+T)=s(t)$ for all $t$. If the signal is piecewise continuous in a period, with finitely many points of discontinuity and finitely many maxima or minima (also known as Dirichlet's conditions), then the Fourier Series converges to $\left[s(t+)+s\left(t^{-}\right)\right] / 2$ (see, for example [14], for details on existence and convergence of the Fourier Series). In that case, the Fourier Series of the signal $s(t)$ is given by

$$
s(t)=A_{0}+\sum_{i=1}^{\infty} A_{n} \cos \left(n \omega_{0} t\right)+\sum_{i=1}^{\infty} B_{n} \sin \left(n \omega_{0} t\right),
$$

with

$$
\begin{gathered}
A_{0}=\frac{1}{T} \int_{\frac{-T}{2}}^{\frac{+T}{2}} s(t) d t, \\
A_{n}=\frac{2}{T} \int_{\frac{-T}{2}}^{\frac{+T}{2}} s(t) \cos \left(n \omega_{0} t\right) d t, \\
B_{n}=\frac{2}{T} \int_{\frac{-T}{2}}^{\frac{+T}{2}} s(t) \sin \left(n \omega_{0} t\right) d t,
\end{gathered}
$$

where $\omega_{0}=2 \pi f$, and $f=1 / T$, the fundamental frequency of $s(t)$.

Fourier series may come in different "flavors and tastes". Here, we are interested in the different connections and interpretations it may have from a future electrical engineer point of view. For example, see chapter 5 of or for the connections between the complex and trigonometric forms of the Fourier Series, the Fourier Series as a special case of the Fourier Integral, waveform sampling, and sampling theorems.

\section{A. Fourier Series of Even, Odd and Half-wave symmetric signals}

The Fourier series expansion of an even signal $s_{e}(t)$ with the period of $T$ does not involve any odd terms and so it has the form

$$
s_{e}=A_{0}+\sum_{n=1}^{\infty} A_{n} \cos \left(n \omega_{0} t\right)
$$

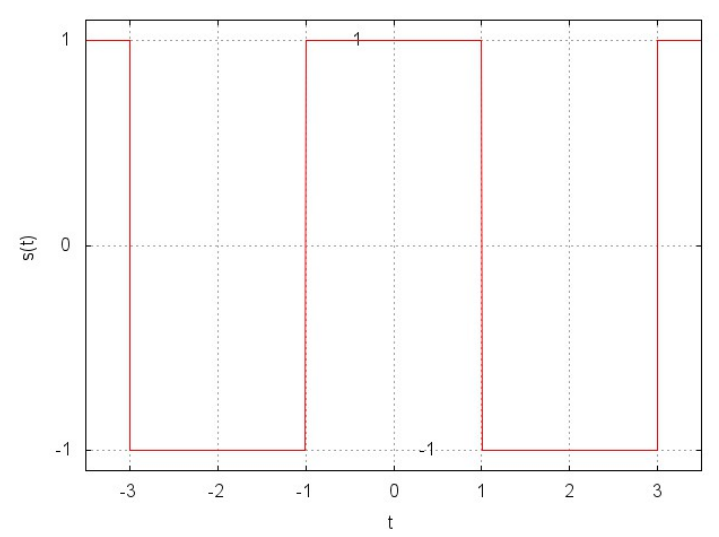

Figure 1. Plot of the square-like wave signal defined by equation 1 .

where the coefficients are given by

$$
A_{0}=\frac{2}{T} \int_{0}^{\frac{+T}{2}} s_{e}(t) d t
$$

and

$$
A_{n}=\frac{4}{T} \int_{0}^{\frac{+T}{2}} s_{e}(t) \cos \left(n \omega_{0} t\right) d t
$$

Similarly, the Fourier Series expansion of an odd $T$ periodic signal $s_{o}(t)$ consists of sine terms only and has the form

$$
s_{o}=\sum_{n=1}^{\infty} B_{n} \sin \left(n \omega_{0} t\right)
$$

where

$$
B_{n}=\frac{4}{T} \int_{0}^{\frac{+T}{2}} s_{o}(t) \sin \left(n \omega_{0} t\right) d t
$$


A $T$-periodic signal $s_{h}(t)$ is said to have half-wave symmetry if $s_{h}(t \pm T / 2)=-s_{h}(t)$. In that case, the Fourier series expansion coefficients will be

$$
\begin{gathered}
A_{0}=0, \\
A_{n}=\left\{\begin{array}{cc}
\frac{4}{T} \int_{0}^{\frac{+T}{2}} s_{h}(t) \cos \left(n \omega_{0} t\right) d t, & \text { n oven, },
\end{array}\right. \\
B_{n}=\left\{\begin{array}{cc}
0, & \text { n even }, \\
\frac{4}{T} \int_{0}^{\frac{+T}{2}} s_{h}(t) \sin \left(n \omega_{0} t\right) d t, & n \text { odd } .
\end{array}\right.
\end{gathered}
$$

\section{B. A typical example-square wave}

The next example shows a typical calculation of the Fourier series expansion coefficients, that we usually solve during the theoretical-practical classes.

Let the periodic signal $s(t)$ be defined by

$$
s(t)=\left\{\begin{array}{cc}
-1, & -2<t<-1 \\
1, & -1<t<1 \\
-1, & 1<t<2
\end{array}\right.
$$

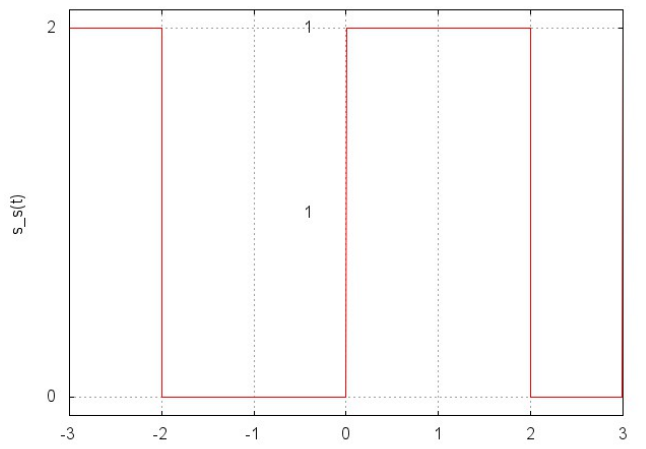

Figure 2. Result of shifting and translating by one the signal presented in equation 1 .

Typically, to find the coefficients of its Fourier series expansion we begin by plotting the signal. One possible result is shown in fig. 1. From this figure (or from equation 1 ) we can easily find that the period is $T=4$, leading to $\omega_{0}=2 \pi / T=\pi / 2$, and that $s(t)=-s(t)$, leading to $B_{n}=0$. Additionally, we may also note that $s(t+T / 2)=-s(t)$ (halfwave symmetry), leading to $A_{n}=0$ for all $\mathrm{n}$ even. In conclusion, we only need to find the coefficients $A_{n}$ for $n$ odd, and so the Fourier Series expansion will be of the form

$$
s=\sum_{\substack{n=1 \\ \text { nodd }}}^{\infty} A_{n} \cos \left(n \frac{\pi}{2} t\right) .
$$

By the definition above,

$$
\begin{gathered}
A_{n}=\frac{4}{T} \int_{0}^{+\frac{T}{2}} s(t) \cos \left(n \omega_{0} t\right) d t, \\
=\frac{4}{T}\left\{\int_{0}^{1} \cos \left(n \omega_{0} t\right) d t+\int_{0}^{2}-\cos \left(n \omega_{0} t\right) d t\right\}, \\
=\frac{4}{T}\left\{\left[\frac{\sin \left(n \omega_{0} t\right)}{n \omega_{0} t}\right]_{0}^{1}-\left[\frac{\sin \left(n \omega_{0} t\right)}{n \omega_{0} t}\right]_{1}^{2}\right\}, \\
=\frac{4}{\frac{2 \pi}{\omega_{0}} n \omega_{0}}\left\{\sin \left(n \frac{\pi}{2}\right)-\sin (n \pi)+\sin \left(n \frac{\pi}{2}\right)\right\}, \\
=\frac{4}{2 n \pi}\left[2 \sin \left(n \frac{\pi}{2}\right)\right],
\end{gathered}
$$

resulting in

$$
A_{n}= \begin{cases}\frac{-4}{n \pi}, & n=3,7,11, \cdots, \\ \frac{4}{n \pi}, & n=1,5,9, \cdots .\end{cases}
$$

Note that the signal plotted in fig. 1 and the corresponding expansion coefficients are the same used in the "square"-like wave form used in the available signals of the application (also illustrated in fig. 3).

Now, suppose that we shift the signal up and translate it right by one. The resulting signal, $s_{s}(t)$, is presented in fig. 2. As a consequence, the signal will no longer be even, and will also lose its half-wave symmetry. Students often

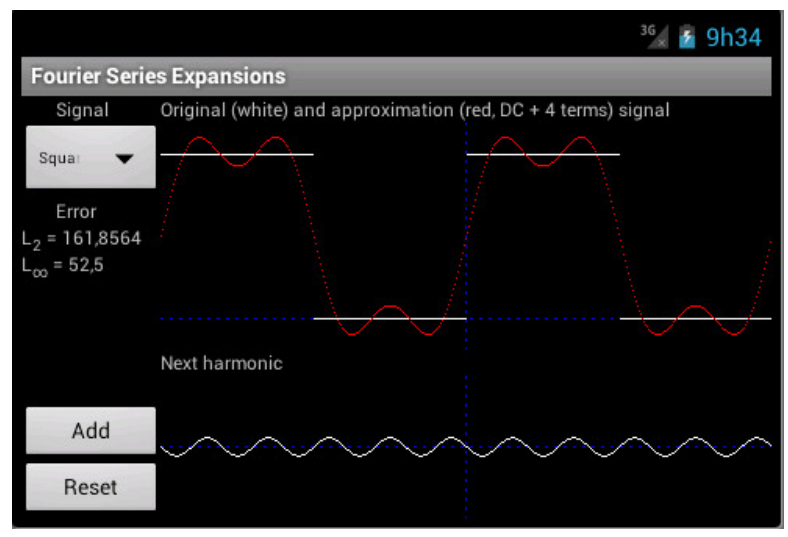

Figure 3. Fourier series expansion of a square-like wave: DC term plus 4 harmonics.

mistakenly assume that the translation converts the signal into an odd one, but the shift up also removes this symmetry (despite of the signal shape, which remains squarelike). In this particular case, the new coefficients will be

$$
\begin{gathered}
A_{0}=1, \\
A_{n}=0, \\
B_{n}=\frac{8}{n \pi}, \quad \text { nodd. }
\end{gathered}
$$

This means that

$$
s_{s}=1+\sum_{\substack{n=1 \\ \text { nodd }}}^{\infty} \frac{8}{n \pi} \sin \left(n \frac{\pi}{2} t\right),
$$

or

$$
s_{s}=1+\frac{8}{\pi} \sin \left(\frac{\pi}{2} t\right)+\frac{8}{3 \pi} \sin \left(3 \frac{\pi}{2} t\right)+\cdots
$$

This type of manipulations (translations and shifts) are made so that students can gain insight on the changes introduced in the coefficients of the Fourier series expansion, and realize how different the new coefficients may become.

\section{THE ANDROID APP}

The Android application, named "FouSE-Fourier Series Expansions", is freely available for download from "Google Play" (www.google.com). Android was our first choice, because the majority of our students have Android powered devices; still, we want to produce an iOS equivalent application. The design of the user interface respects the guidelines discussed for example in [5, 7]. So, the "control section" is located in the left side of the interface, in the right-upper half the original signal and 
the approximation signal are plotted, and in the rightlower half the next term (harmonic) to be added is plotted. Fig. 3 shows a screenshot of the application. When no signal is selected, the spinner on the upper left corner will show the word "none" and the plotting spaces of the original and approximation signals, as well as the next harmonic plot, will be empty, because they have nothing to show.

The user interaction with the application is very straightforward. The user begins by choosing a signal from

the list (by pressing the spinner below the text "Signal"). Then, by simply pressing the "Add" button, the user refines the approximation to the desired accuracy.

The list of available signals, and whose analytical Fourier series coefficients are found during the theoretical lectures, includes:

- Sawtooth;

- $\quad|\sin |$ (full wave rectified);

- Half $\sin$ (half wave rectified);

- Square;

- Triangle;

- Parabolic $\left(t^{2}\right)$.

As stated above, the "control" section of the interface is placed on the left side of the screen. As seen above, the spinner below the word "Signal" is used to choose the signal to approximate. Once a signal is chosen, more information and control buttons will be visible. The error of the approximation over a period is presented using two different metrics:

$$
\begin{array}{ll}
\text { - } & L_{2}=\sqrt{\sum_{i=1}^{N}\left(\text { signal }_{i}-\text { approximation }_{i}\right)^{2}} ; \\
\text { - } & L_{\infty}=\max _{i} \mid \text { signal }_{i}-\text { approximation }_{i} \mid .
\end{array}
$$

The "Add" button is used to add the next term (the signal presented next to this button and below the text "Next harmonic") to the approximation signal. The "Reset" button recomputes the approximation.

The upper right side of the screen presents two signals plots and one text message:

- the original signal (in white);

- the current approximation (in red);

- the number of terms used in the current approximation (DC term, plus harmonics, if any).

Every time the "Add" button is pressed, the current harmonic plotted below the text "Next harmonic" is added to the current approximation, a new harmonic is presented here replacing the previous one, the approximation signal is re-plotted, the new errors are computed, and the number of terms used in the current approximation is updated in the text message next to the main graph.

Fig. 3 shows an example of an approximation. In this case, the original signal is a square-like wave, plotted in white, and the result of the approximation, after summing the DC component plus three harmonics/terms of the Fourier series expansion, is plotted in red, over the same graph. On the graph below, it is presented the plot of the next harmonic/term to be added to the result of the approximation plotted in red, if the "Add" button is pressed. The current error of the approximation over a period is also shown $\left(L_{2}=161.8564\right.$ and $\left.L_{\infty}=52.5\right)$, and will decrease as more harmonics/terms are added to the approximation.

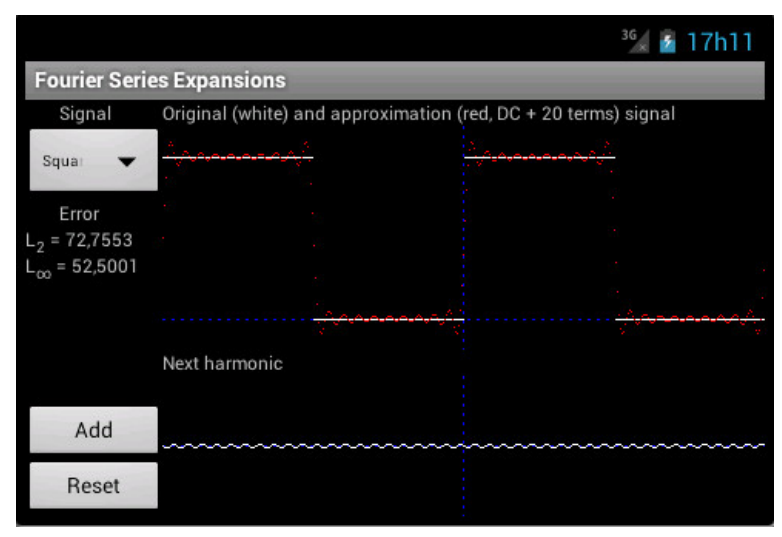

Figure 4. Fourier series expansion of a square-like wave: DC term plus 20 harmonics.

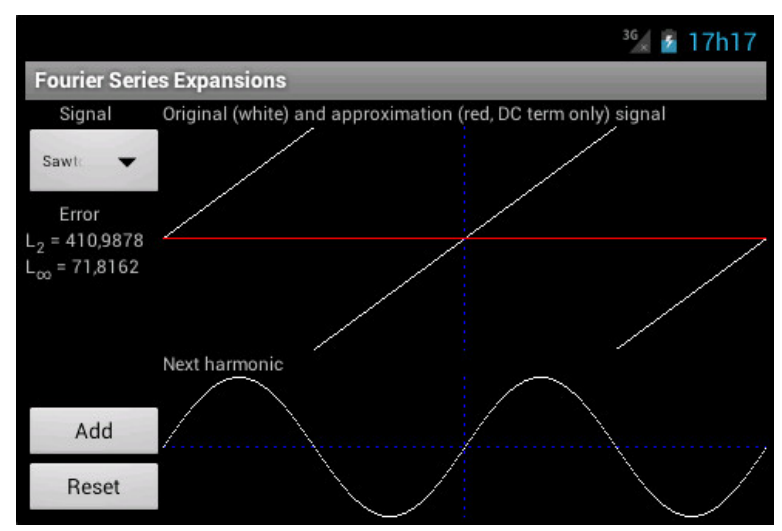

Figure 5. Fourier series expansion of a sawtooth-like wave: DC term only.

Note that the scales (and axes) used for the signals and harmonic plots are different, and automatically adapt from expansion to expansion. However, these scales remain constant during a particular expansion, so the student can realize the different weights that successive harmonics will have in the approximation. For example, fig. 4 shows the result of the Fourier series approximation to a squarelike wave, after using the DC term plus 20 harmonics. When compared to fig. 3 we may note the big difference in the weights of harmonic number 4 and harmonic number 21 (obviously, the same applies to the error measures $L_{2}=72.7553$ and $\left.L_{\infty}=52.5\right)$. Note that, in this case, $\mathrm{L}_{\infty}$ error remains constant $(52.5)$; this is due to the Gibbs phenomenon (see, for example, for details).

Figures 5 and 6 show the Fourier series expansion approximation to a sawtooth-like wave. Fig. 5 depicts an approximation consisting of the DC term only (all the approximations start with the DC term only). In this case it is clear that $\mathrm{DC}=0$ and so it overlaps the $\mathrm{t}$ (time) axis in the plot. Also note that the first harmonic term was scaled to completely fill the plotting area reserved to the plot of the harmonics. Now, from fig. 6 we can see that the fifth harmonic term will have a much lower weight in the series' final result. Note also that the time axis is now well visible in the approximation plot; as stated above, when the approximation had only the DC term, it overlapped the time axis.

Fig. 7 shows a screenshot of the help menu, available by pressing the "menu" button of the smartphone. This menu file consists of three topics, a "quick start", an explanation of the interface, and a brief introduction to the Fourier series. 


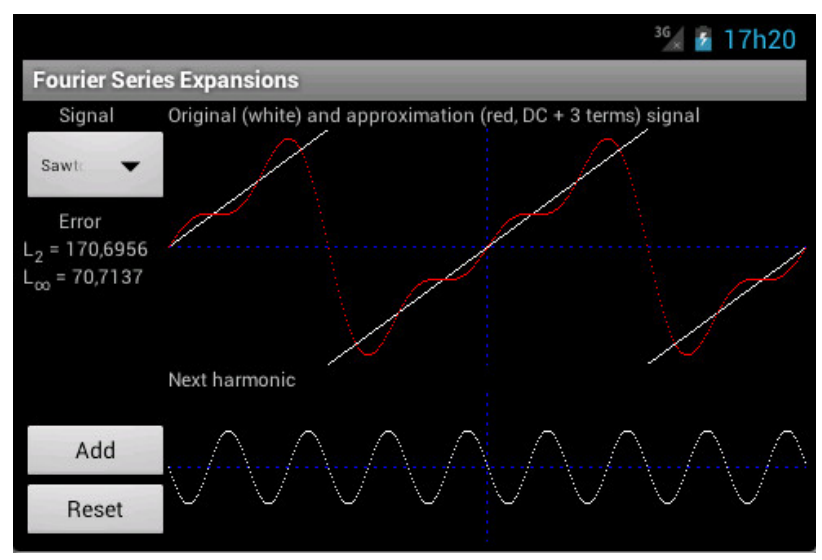

Figure 6. Fourier series expansion of a sawtooth-like wave: DC term plus four harmonics.

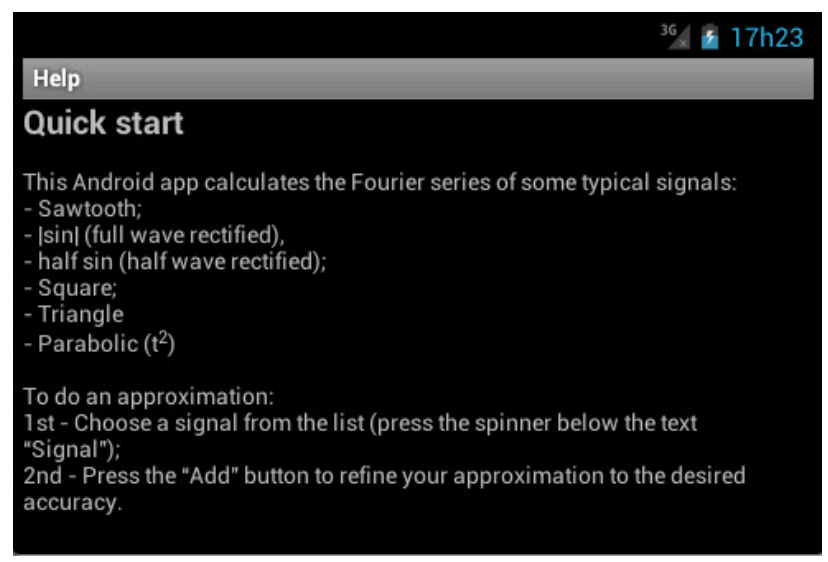

Figure 7. Screenshot of the help menu, showing the "quick start" topic.

\section{SURVEY AND RESULTS}

We have distributed a copy of the application directly to our students, i.e., the students installed the app directly on their devices without the need of downloading it from "Google Play", after the subject was taught in class. The students were anonymously asked the following questions:

1. Gender.

2. Do you consider that the application has an ease to use interface?

3. Have you ever used or needed to use the help menu?

4. Do you prefer a web-based tool (applet) or this (android) version? Please tell us why.

5. Do you think that the application matches/covers the contents taught related to the Fourier series?

6. Do you think this application helped you in understanding the Fourier series (basics, how it works and its applicability)?

7. Do you think this application helped you in understanding other topics/subjects in this or other courses?

8. Do you think it would be beneficial to have similar tools in other subjects \& courses?

From a total of 85 enrolled students, 55 completed the survey, from which $50(91 \%)$ were males and $5(9 \%)$ females. $51(93 \%)$ of the surveyed students consider that the app has an ease to use interface, and only 4 (7\%) found it somehow difficult or hard to use. However, only $2(4 \%)$ of the total responding students have tried the help menu; curiously, these students are two of the four who considered the application hard or difficult to use.

From the responding students, $47(85 \%)$ prefer this Android version, when compared to other web-based applications (applet like) available on the Internet. When asked why, they answered that this application "is really portable (in the sense that we don't need to be always connected; we can do simulations even if we do not have Internet access)" and "it seems that the interface was designed and developed to extract the maximum from my android device, in contrast to a web-based applet were we constantly need to adjust the screen size, resolution, among other". The other $8(15 \%)$ students consider the web-based (applet) version more suited to their needs: "don't like to install apps" and "web-based applications are really platform independent" were the two main reasons presented for that. In this group we also counted the iOS (iPhone/iPad) users, who revealed that they are willing for an iOS version of the tool.

A total of $53(96 \%)$ of the responding students consider that the tool matches/covers the subjects studied within the Fourier Series topics, and 54 (98\%) believe that it helped them in understanding the basics of the Fourier Series, how it works and its applicability. In addition, 50 $(91 \%)$ students consider that this tool helped them to understand other topics under this course and even in other courses. The referred topics include: some signals' properties (like symmetry, frequency, etc.); Fourier Transform; math series construction; and programming (the code of the application was made available to our students).

When asked if other courses/topics/subjects should benefit from similar tools/applications the answer was unanimous: $100 \%$ (55) of the responding students said yes.

\section{CONCLUSIONS}

Fourier series expansions are among the problems most often faced by an Electrical Engineer. An undergraduate course in the subject must provide the student with the relevant know-how, and it may also be of considerable value in understanding how a background in Fourier and numerical analysis can be applied to concrete engineering problems.

In this context, we felt a need for tools that could be easily used inside or outside the classroom, in formal and informal contexts, and at the same time were easy to update and maintain. The application that we have presented and described allows students to try several Fourier series expansions on a set of typical signals, and then compare the results in an easy-to-use environment, and using their own Android powered devices.

The application, named "FouSE-Fourier Series Expansions", is freely available for download from "Google Play" (www.google.com), and can be used by the students in their daily work, inside or outside the classroom, and (as reported by the sudents themselves) has proved to be a valuable tool for teaching and understanding Fourier series expansions.

\section{REFERENCES}

[1] C. O’Malley, G. Vavoula, J. Glew, J. Taylor, M. Sharples, and P. Lefrere, "WP4 - guidelines for learning/teaching/tutoring in a mobile environment," MOBIlearn/UoN, UoB, OU, Tech. Rep., 2003. 
[2] P. Harris, "Going mobile. Learning Circuits," ASTD Online Magazine. http://www.astd.org/, 2001.

[3] M. Sharples, "The design of personal mobile technologies for lifelong learning," Computers \& Education, vol. 34, no. 3-4, pp. 177-193, 2000. [Online]. Available: http://www.science direct.com/science/article/pii/S0360131599000445

[4] J. Moore, S. Oussena, and P. Zhang, "A portable document search engine to support off-line mobile learning," in IADIS International Conference Mobile Learning 2009, February 2009, event Dates: 26th, $2009 . \quad$ [Online]. Available: http://eprints.soton.ac.uk/267441/

[5] A. Holzinger, M. Geier, and P. Germanakos, "On the development of smart adaptive user interfaces for mobile e-Business applications: Towards enhancing user experience - some lessons learned," in Proceedings of the International Conference on eBusiness (ICE-B), Rome, Italy, 2012, pp. 3-16.

[6] T. Elias, "Universal instructional design principles for mobile learning," The International Review of Research in Open and Distance Learning, vol. 12, no. 2, 2011. [Online]. Available: http://www.irrodl.org/index.php/irrodl/article/view/965

[7] S. Mujacic, M. Debevc, P. Kosec, M. D. Bloice, and A. Holzinger, "Modelling, design, development and evaluation of a hypervideo application for digital systems teaching," Multimedia Tools and Applications, no. 2, pp. 435-452, 2012. http://dx.doi.org/ 10.1007/s11042-010-0665-1

[8] A. Holzinger, A. Nischelwitzer, S. Friedl, and B. Hu, "Towards life long learning: Three models for ubiquitous applications," Wireless Communications and Mobile Computing, no. 10, pp. 1350-1365, 2010

[9] H.-C. Chu, G.-J. Hwang, S.-X. Huang, and T.-T. Wu, "A knowledge engineering approach to developing e-libraries for mobile learning," Electronic Library, vol. 26, no. 3, pp. 303-317, 2008, Workshop on Language and Cultural Change, Groningen, Netherlands, Feb, 2005.

[10] G.-J. Hwang and H.-F. Chang, "A formative assessment-based mobile learning approach to improving the learning attitudes and achievements of students," Computers \& Education, vol. 56, no. 4, pp. 1023-1031, May 2011. http://dx.doi.org/10.1016/j.compedu. 2010.12.002

[11] E. Martin and R. M. Carro, "Supporting the Development of Mobile Adaptive Learning Environments: A Case Study," IEEE Transactions on Learning Technologies, vol. 2, no. 1, pp. 23-36, Jan-Mar 2009. http://dx.doi.org/10.1109/TLT.2008.24

[12] S. Nooshabadi and J. Garside, Modernization of teaching in embedded systems design - An international collaborative pro- ject," IEEE Transactions on Education, vol. 49, no. 2, pp. 254262, MAY 2006. http://dx.doi.org/10.1109/TE.2006.872402

[13] M. J. C. S. Reis, S. Soares, C. Sim ao, R. Morais, E. Soares, and P. J. S. G. Ferreira, "Teaching of fourier series expansions in undergraduate education," in Proceedings of the IEEE International Conference EDUCON 2013, 13th-15th March, Berlin, Germany, 2013, p. Accepted for publication.

[14] G. P. Tolstov, Ed., Fourier Series. N.Y.: Dover Publications Inc., 1976.

[15] E. O. Brigham, The Fast Fourier Transform. Englewood Cliffs, NJ: Prentice-Hall, 1974.

[16] A. Ambardar, Analog and digital signal processing. Boston, MA: PWS Publishing Company, 1995.

[17] A. J. Jerri, Ed., Advances in the Gibbs Phenomenon. Sampling Publishing, 2011.

\section{AUTHORS}

M. J. C. S. Reis is with the Engineering Department, University of Trás-os-Montes e Alto Douro (e-mail: mcabral@utad.pt).

S. Soares is with the Engineering Department, University of Trás-os-Montes e Alto Douro (e-mail: salblues@utad.pt).

S. Cardeal is with the Portugal Telecom Inovação (email: simao-o-cardeal@ptinovacao.pt).

R. Morais is with the Engineering Department, University of Trás-os-Montes e Alto Douro (e-mail: rmorais@utad.pt).

E. Peres is with the Engineering Department, University of Trás-os-Montes e Alto Douro (e-mail: eperes@utad.pt).

P. J. S. G. Ferreira is with the Department of Electronics, Telecommunications and Informatics (DETI), University of Aveiro (e-mail: pjf@ua.pt).

${ }^{*}$ This work was supported in part by Portugal Telecom Inovação in the context of the project e-VoIP.

This article is an extended and modified version of a paper presented at the 5th International Conference on Computer Supported Education (CSEDU 2013), 6-8 May 2013, Aachen, Germany. Submitted 01 December 2013. Published as re-submitted by the authors 05 January 2014. 